\title{
TOMOGRAPHIC RECONSTRUCTION OF VELOCITY ANOMALIES
}

\author{
By John A. Fawcett and Robert W. Clayton
}

\begin{abstract}
An approximate inversion formula is proposed for the reconstruction of slowness anomalies in a known depth varying background field. The data are observed travel-time perturbations for reflections from a known planar reflector. The limitations of the formula are discussed and numerical examples are given.
\end{abstract}

\section{INTRODUCTION}

Tomography refers to the technique of reconstructing a field from line or surface integrals of it. In medical X-ray tomography, for example, the tissue density field is deduced from measurements of $\mathrm{X}$-ray attenuation through the patient. In this case the data are regularly sampled line integrals, so they are discrete values of a Radon transform.

In seismology, the determination of slowness (inverse of velocity) and attenuation fields can also be viewed in a tomographic framework. The travel times or the amplitude decay along rays connecting the sources and receivers are the projections of the fields. Here, the problem is complicated by the fact that the rays are curved and that the ray path depends on the slowness field itself. The problem is in general nonlinear. One can linearize the problem about a reference slowness which essentially decouples the ray paths from the unknown slowness field. However, this leaves the problem of tomographic reconstruction from line integrals along curved rays.

Our methods discussed below will be thought of in terms of a reflection seismology experiment. However, the results are also applicable to transmission problems. The goal of our tomographic reconstruction is to identify from travel-time information (source positions known) areas of relatively high and slow velocity (with respect to a known background field) within a layer of the earth.

We examine the theory of tomographic reconstruction when the reference slowness is taken to be a known function of depth. Also, although we specialize the problem to depth-dependent background velocities and flat reflectors, we hope that our results for this case will indicate the concepts to apply for more general situations. The generalized Inverse Radon Transform which we will derive for curved ray projections is similar to that derived independently by G. Beylkin (1982).

\section{Linearization of the Forward Problem}

The travel time, between a source $x_{s}$ and receiver $x_{r}$, along the ray path, $r\left(x_{s}, x_{r}\right)$, can be written as

$$
t\left(x_{s}, x_{r}, n\right)=\int_{r a y} n\left[r\left(x_{s}, x_{r} ; n\right)\right] d s
$$

In (1) $n$ is the slowness, and $d s$ is the differential arclength. The expression (1) is nonlinear in $n$. To decouple the ray paths $r\left(x_{s}, x_{r} ; n\right)$ from the unknown slowness field, $n$, we write the slowness as a perturbation about an assumed reference field $n_{0}$.

$$
\begin{gathered}
n(\vec{x})=n_{0}(\vec{x})+\Delta n(\vec{x}) . \\
2201
\end{gathered}
$$


If we consider $\Delta t\left(x_{s}, x_{r}\right)$ to be the resultant perturbation of the travel-time $t\left(x_{s}, x_{r} ; n\right)$, due to the perturbation (2), we can write

$$
\Delta t\left(x_{s}, x_{r}\right)=\int_{r a y} \Delta n\left(r\left(x_{s}, x_{r} ; n_{0}\right)\right) d s+O\left(\Delta n^{2}\right) .
$$

The simplicity of expression (3) is due to the fact that the variation of the travel time with respect to the change in ray path is zero from Fermat's principle. Thus, the principle, which is fundamental to linearized travel-time analysis, is: the perturbation of the travel time between $x_{s}$ and $x_{r}$ is to first order the integral of the perturbation of the slowness field along the unperturbed ray.

\section{InVersion for a Depth Varying Background Slowness Field}

In this paper we will specifically consider a reflection or backscatter geometry. However, we will convert this problem into an equivalent transmission problem before deriving our tomographic inversion formula. Hence, the concepts presented can be readily applied to transmission problems. The geometry is shown in Figure 1.

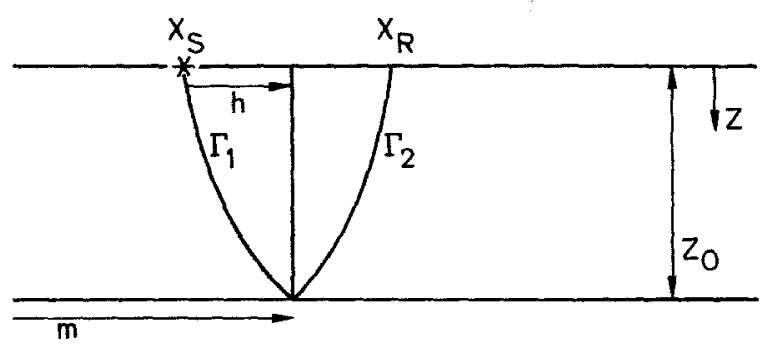

FIG. 1. Geometry and notation for reflection travel times.

We will denote the travel time from a source, $x_{s}$, to receiver, $x_{r}$, as $t(m, h)$ where $m$ is the midpoint, $m=\left(x_{s}+x_{r}\right) / 2$ and $h$ is the offset, $h=\left(x_{r}-x_{s}\right) / 2$. In these coordinates, equation (3) is

$$
\Delta t(m, h)=\int_{r(m, h)} \Delta n(\vec{x}) d s .
$$

Our goal is the inversion, or approximate inversion, of (4) to determine the field, $\Delta n$, from the field $\Delta t(m, h)$. Here $r(m, h)$ denotes the ray path for the mid-point $m$ and offset $h$.

For a slowness field, $n_{0}(z)$, we can write analytic expressions for the two ray segments $\Gamma_{1}(z ; m, h)$ and $\Gamma_{2}(z ; m, h)$, shown in Figure 1 . On the descending ray segment, $\Gamma_{1}$, we write

$$
x=m-q(z, h)
$$

and on the ascending ray segment, $\Gamma_{2}$

$$
x=m+q(z, h)
$$


where

$$
q(z, h)=\int_{z}^{z_{0}} \frac{p(h) c(y)}{\sqrt{1-p(h)^{2} c(y)^{2}}} d y
$$

In this notation, $p(h)$, is the ray parameter for a given offset $h\left(p \equiv \cos \Theta_{0} / c(0)\right.$, where $\Theta_{0}$ is the take-off angle of the ray at the source).

An equivalent model to that shown in Figure 1 is obtained by reflecting the layer and its slowness field about the line $z=z_{0}$ as is shown in Figure 2. This new geometry allows the ray path to be expressed as

$$
x(z)=m-\int_{0}^{z_{0}} \frac{p(h) c(y)}{\sqrt{1-p(h)^{2} c^{2}}} d y .
$$

For equation (4), we write

$$
\Delta t(m, h)=\int_{0}^{2 z_{0}} \Delta n(x-q(y, h), y) \frac{d s}{d z}(y, h) d y .
$$

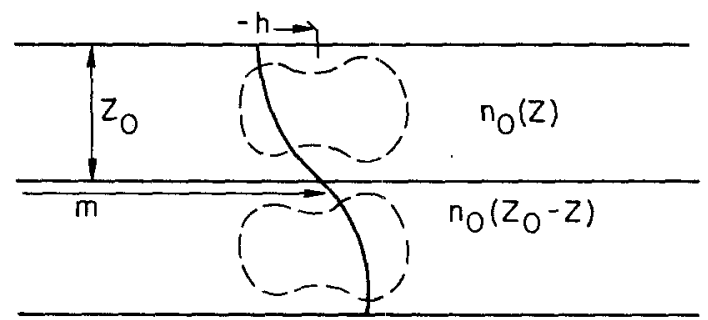

FIG. 2. Equivalent transmission geometry for symmetrized field.

For a constant background velocity the rays are straight lines. Thus in (9), $\Delta t$ is simply the standard two-dimensional Radon Transform of $\Delta n$ expressed in terms of mid-point and offset. Hence, an exact inversion formula (at least in a domain of Fourier space) can be found. For a depth-dependent background field, the rays for small offsets are only slightly curved, and using an expansion of the integrand of (9) about $h=0$, and using an appropriate change of variables, we can once again put (9) into the form of the standard Radon Transform. The inversion formulas for the above problems are given in the sections on "Small-Offset Approximation" and "Constant Background Velocity", respectively.

These two cases lead one to consider a backprojection approximation to $\Delta n(x, z)$. That is, to reconstruct the slowness field, $\Delta n(x, z)$ at a point $(x, z)$ we composite weighted travel-time perturbations that correspond to rays which pass through $(x, z)$. As we shall now show, a good backprojection formula to consider is

$$
n_{1}(\bar{x}, \bar{z})=\int_{-h_{\max }}^{h_{\max }} \frac{\Delta t(\bar{x}+q(\bar{z}, h), h)}{\frac{d s}{d z}(\bar{z}, h)}\left|\frac{\partial^{2} q}{\partial z \partial h}\right| d h .
$$

Each travel-time contribution is divided by the local arclength, $d s / d z(\bar{z}, h)$, and 
multiplied by a ray focussing factor, $\left|\left(\partial^{2} q / \partial z \partial h\right)(\bar{z}, h)\right|$. The limits of integration, $\pm h_{\max }$, emphasize the fact that we can observe $\Delta t(m, h)$ for only a finite range of offsets. Also, we are only interested in those rays that do not have turning points within the layer.

To show the validity of equation (10), we substitute (9) into (10) to obtain

$$
\begin{aligned}
n_{1}(\bar{x}, \bar{z})=\int_{-h_{\max }}^{h_{\max }} \int_{0}^{2 z_{0}} \Delta n(\bar{x}+q(\bar{z}, h)-q(z, h), z) \\
\\
\qquad \frac{\frac{d s}{d z}(z, h)}{\frac{d s}{d z}(\bar{z}, h)}\left|\frac{\partial^{2} q(\bar{z}, h)}{\partial z \partial h}\right| d z d h .
\end{aligned}
$$

We now consider a polar coordinate system centered at $(\bar{x}, \bar{z})$, which we show diagramatically in Figure 3. Mathematically, the domain $\Omega$ of Figure 3 is

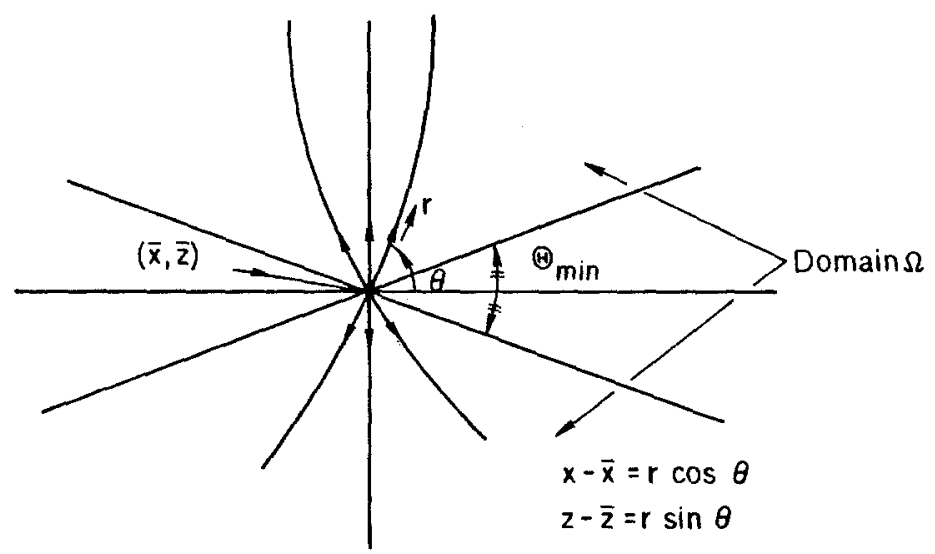

FIG. 3. Ray/polar coordinate system.

$\left((x, z)|| z / x \mid>\tan \Theta_{\min }\right)$. We now change variables from $(h, z)$ to $(\Theta, r)$ where

$$
\begin{aligned}
\Theta & =-\cot ^{-1}\left(\frac{\partial q}{\partial z}(z, h)\right) \\
\frac{d s}{d z} & =\frac{1}{\sin \Theta} ; x-\bar{x}=r \cos \Theta z-\bar{z}=r \sin \Theta .
\end{aligned}
$$

The Jacobian of this above change variables is

$$
\left|\frac{\partial(h, z)}{\partial(\Theta, r)}\right|=\left|\frac{\partial^{2} q}{\partial z \partial h}(\bar{z}, h) \sin \Theta\right|^{-1} \text {. }
$$

We can now write (11) as

$$
n_{1}(\bar{x}, \bar{z})=\int_{\mathrm{a}} \frac{\Delta n\left(\bar{x}+r \cos \Theta+R_{1}(\Theta, r), \bar{z}+r \sin \Theta\right)\left(1+R_{2}(\Theta, r)\right) r d r d \Theta}{|z-\bar{z}|} .
$$


The terms $R_{1}(\Theta, r)$ and $R_{2}(\Theta, r)$ are remainder terms from the first order Taylor expansion of the integrand in (14) about $z=\bar{z}$. We now assume that a good approximation to (14) is a local approximation, where we let the $z$ limits of $\Omega$ go to $\pm \infty$

$$
n_{1}(\bar{x}, \bar{z})=\int_{\Omega} \frac{\Delta n(\bar{x}+r \cos \Theta, \bar{z}+r \sin \Theta) r d r d \Theta}{|z-\bar{z}|}
$$

We can write (15) in the form

$$
n_{1}(\bar{x}, \bar{z})=\Theta(\bar{x}, \bar{z}) * \Delta n(\bar{x}, \bar{z})
$$

where $\Theta(x, z)$ has the form

$$
\begin{aligned}
\Theta(x, z) & =\frac{1}{|z|} \text { for }\left|\frac{z}{y}\right| \geqq \tan \Theta_{\min } . \\
& =0 \text { otherwise. }
\end{aligned}
$$

Formal calculations show that in the Fourier domain, $\tilde{\Theta}\left(k_{x}, k_{z}\right)$ has the following form

$$
\begin{aligned}
\tilde{\Theta}\left(k_{x}, k_{z}\right) & =\frac{1}{\left|k_{x}\right|} \text { for }\left|\frac{k_{z}}{k_{x}}\right| \leq \cot \Theta_{\min } \\
& =0 \text { otherwise. }
\end{aligned}
$$

Equations (15) and (16c) suggest the following improvement to the back-projection formula (10)

$$
n_{1}(\bar{x}, \bar{z})=F(\bar{x}) * \int_{-h_{\max }}^{h_{\max }} \frac{\Delta t(\bar{x}+q(\bar{z}, h), h)}{\frac{d s}{d z}(\bar{z}, h)}\left|\frac{\partial^{2} q}{\partial z \partial h}\right| d h .
$$

Here, $F(x)$ is defined formally as $F(x)=F^{-1}\left(\left|k_{x}\right|\right)$. In practice, $F(x)$ will be the inverse Fourier transform of some finite approximation to $\left|k_{x}\right|$. The filter $F(x)$ is proportional to the Hilbert Transform of the $d / d x$ operator. Intuitively, as the backprojected field is an integral (a smoothing operator) over all travel-time perturbations from rays passing through the point $(\bar{x}, \bar{z})$, it is clear some type of high-frequency operation must be applied to the back-projection approximation to recover the true field. The numerical implementation of the filter is described below in "The Inversion Program" [see equation (26)]. Equation (17) is the generalized back-projection formula we will employ. Below, we give simplifications of (17) for various special cases.

\section{SMALL-OFFSET APPROXIMATION}

Over the range of integration in (17), $h \in\left[-h_{\max }, h_{\max }\right]$, we will expand the integrand in a first order Taylor series about $h=0$. Thus, we will use the following 
expansions

$$
\begin{aligned}
q(\bar{z}, h) & =\frac{\int_{z}^{z_{0}} c(y) d y}{\int_{0}^{z_{0}} c(y) d y}+O\left(h^{2}\right) \\
\left|\frac{\partial^{2} q}{\partial z \partial h}\right|^{h=0} & =\frac{c(\bar{z})}{\int_{0}^{z_{0}} c(y) d y}+O\left(h^{2}\right) \\
\left.\frac{d s}{d z}\right|_{h=0} & =1+O\left(h^{2}\right) .
\end{aligned}
$$

We will use the definitions, $\tau \equiv \int_{0}^{\bar{z}} c(y) d y, \tau_{0} \equiv \int_{0}^{z_{0}} c(y) d y$, and now we can write for (17)

$$
n_{1}(\bar{x}, \bar{z})=F(\bar{x}) * \frac{c(\bar{z})}{\tau_{0}} \int_{-h_{\max }}^{h_{\max }} \Delta t\left(\bar{x}+\left(1-\frac{\tau}{\tau_{0}}\right) h, h\right) d h .
$$

\section{Constant Background Velocity}

For a constant background velocity, we have the following relations

$$
\begin{aligned}
q(\bar{z}, h) & =\left(z_{0}-\bar{z}\right) \frac{h}{z_{0}} \\
\frac{d s}{d z} & =\frac{\sqrt{h^{2}+z_{0}^{2}}}{z_{0}} \\
\frac{\partial^{2} q}{\partial z \partial h} & =-z_{0} .
\end{aligned}
$$

Thus (11) becomes, exactly,

$$
n_{1}(\bar{x}, \bar{z})=\int_{-h_{\max }}^{h_{\max }} \int_{0}^{2 z_{0}} \Delta n\left(\bar{x}+(y-\bar{z}) \frac{h}{z_{0}}, y\right) z_{0} d y d h .
$$

Using (20) and the change of variables to polar coordinates (12), we obtain exactly

$$
n_{1}(\bar{x}, \bar{z})=\Theta(\bar{x}, \bar{z}) * \Delta n(\bar{x}, \bar{z}) .
$$

Here, $\cot \Theta_{\min }=h_{\max } / z_{0}$.

Hence, for this case, we can write exactly,

$$
n_{1}(\bar{x}, \bar{z})=\frac{F(\bar{x})}{z_{0}} * \int_{-h \max }^{h_{\max }} \frac{\Delta t\left(\bar{x}+\frac{h}{z_{0}}\left(z_{0}-\bar{z}\right), h\right)}{\sqrt{h^{2}+z_{0}^{2}}} d h
$$


where

$$
\begin{aligned}
\tilde{n}_{1}\left(k_{x}, k_{z}\right) & =\Delta \tilde{n}\left(k_{x}, k_{z}\right) \text { for }\left|\frac{k_{z}}{k_{x}}\right| \leq \frac{h_{\max }}{z_{0}} \\
& =0 \text { otherwise. }
\end{aligned}
$$

These formulas, (23) and (24), for constant background velocity were derived in a different fashion by Kjartansson (1980) and Fawcett (1983).

\section{DePth AND the Relative Resolution}

For the background field $a$ constant, the ratio of maximum offset to the reflector depth, $h_{\max } / z_{0}$, determines from (24) how well we can reconstruct the unknown field in Fourier space. For a depth varying background field, the analog of $h_{\max } / z_{0}$ is the maximum slope, $d x / d z(z)$, of any ray that passes through a point $(x, z)$. For a background field which increases with depth, the slopes of the rays increases with depth. Hence, intuitively, we expect our reconstruction to improve with depth for an increasing background velocity.

More physically, for straight rays, it is clear that with only a knowledge of the

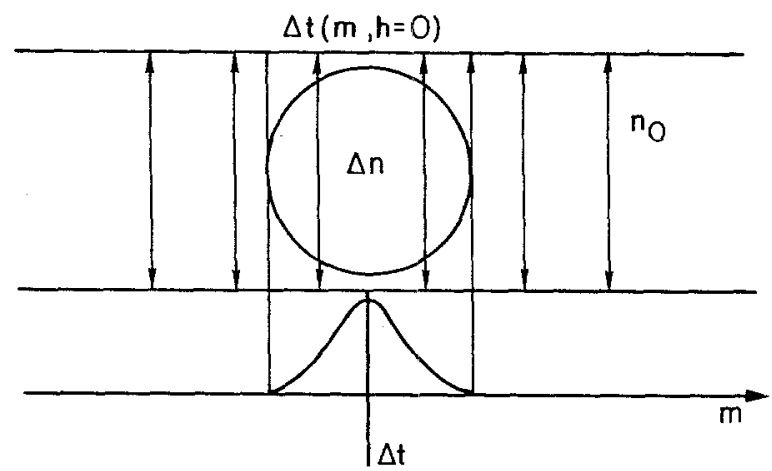

FIG. 4. Example of rays' resolution.

projections along rays, all with the same slope, it is not possible to resolve variations in the unknown field in the direction of the rays. As an example of this statement, consider the geometry shown in Figure 4. We see that for the anomaly and rays of Figure 4, we can determine only the lateral extent of the circle. For seismic experiments, where we have only a finite maximum offset, we deduce that the lack of large offset data will correspond to problems in the vertical resolution of the anomaly. For a depth increasing background velocity field, we expect the vertical resolution of the anomaly to improve, for in this case the effective slopes of the rays increase with depth.

\section{NUMERICAL EXPERIMENTS}

To test our ideas on tomographic inversion of travel-time anomalies, we require two computer programs: one to generate synthetic travel-time perturbations for known anomaly and background fields, and second, an inversion program using either (17), (19), or (23) to invert the travel-time data. All computations were done in single precision Fortran-77 on a VAX computer.

Generation of synthetic data. The data are generated from the projection of the unperturbed ray through the anomaly field. Hence, the data does not correspond 
exactly to the perturbations which would be measured in a true "seismic" experiment. However, as discussed above, this is a first-order accurate approximation. Thus, our numerical examples below test only the tomographic inversion formulas with the assumption that the linearized problem is valid.

We take the background velocity field to be of the form $c(z)=a z+b$. The velocity anomalies are taken to be disks. The disks' radii and position and the value of the constant perturbation within the disk are user input parameters. As mentioned above, we are assuming for the data generation that the linearization assumption (3) is valid. Hence, we can use simple units (e.g., 1, 2, 0.5, etc.) for the perturbation strength within the disks, as it is only the relative size of the perturbations that is relevant. Of course, in reality, the linearization is only valid for $\Delta n$ sufficiently small.

The rays for the field $c(z)=a z+b$ are arcs of circles. Some algebra allows one to determine the equation of these circles for a given offset, $h$, and mid-point $m$. The intersection points (if there are any), $\vec{x}_{1 i}(m, h)$ and $\vec{x}_{2 i}(m, h)$, of the ray with the $i$ th disk are found by applying a quadratic formula. Then, the contribution of the $i$ th disk to the travel-time perturbation, $\Delta t(m, h)$ is $n_{i} s_{i}$, where $n_{i}$ is the constant slowness perturbation within the $i$ th disk, and $s_{i}$ is the arc length of the ray, in the

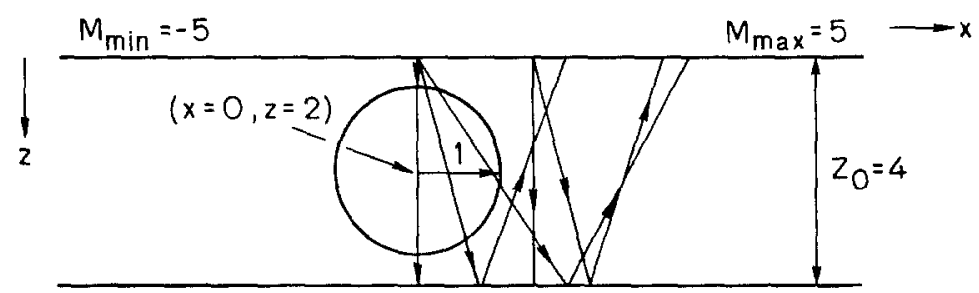

FIG. 5. Geometry for example 1.

disk, between $\vec{x}_{1 i}(m, h)$ and $\vec{x}_{2 i}(m, h)$. This calculation is carried out for all the disks, and for both the descending and ascending ray segments.

The program user specifies the minimum and maximum mid-point, $m_{\max }$ and $m_{\text {min }}$, and the percentage of the maximum offset to calculate $\Delta t(m, h)$ for. The maximum offset, for a velocity profile that increases with depth, is the offset of the ray that has a turning point at $z=z_{0}$.

Sixty-four increments in $h$ and $m$ are then calculated. We only calculate $h>0$ as we know that $\Delta t(m,-h)=\Delta t(m, h)$. This data file is then stored as the input for the inversion program.

The inversion program. The two basic formulas we wish to examine numerically are equations (17) and (19) [and (23) which is (19) and (17) for $c(z) \equiv b$ ]

$$
\begin{aligned}
& n_{1}(\bar{x}, \bar{z})=F(\bar{x}) * \int_{-h_{\max }}^{h_{\max }} \frac{\Delta t(\bar{x}+q(\bar{z}, h), h)}{\frac{d s}{d z}(\bar{z}, h)}\left|\frac{\partial^{2} q}{\partial z \partial h}\right| d h \\
& n_{1}(\bar{x}, \bar{z})=F(\bar{x}) * \frac{c(\bar{z})}{\tau_{0}} \int_{-h_{\max }}^{h_{\max }} \Delta t\left(\bar{x}+\left(1-\frac{\tau}{\tau_{0}}\right) h, h\right) d h .
\end{aligned}
$$


In fact, we do not consider negative offsets but write, e.g., for (17)

$$
n_{1}(\bar{x}, \bar{z})=F(\bar{x}) * \int_{0}^{h_{\max }}\left(\frac{\Delta t(\bar{x}+q(\bar{z}, h), h)+\Delta t(\bar{x}-q(\bar{z}, h), h)}{\frac{d s}{d z}(\bar{z}, h)}\right)\left|\frac{\partial^{2} q}{\partial z \partial h}\right| d h .
$$

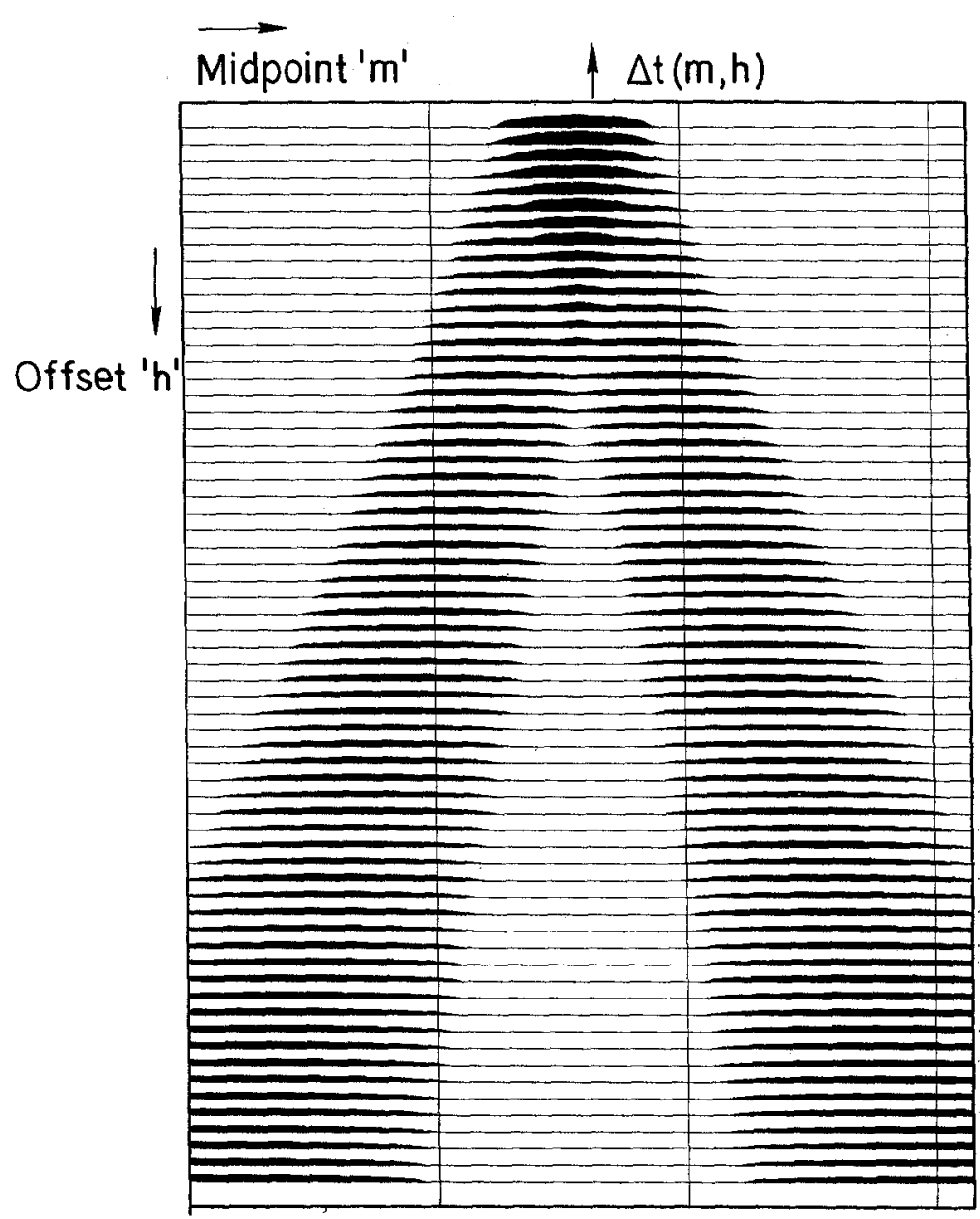

FIG. 6. Travel-time data, $\Delta t(m, h)$, for Figrue $5, c(z) \equiv 1$.

A trapezoidal rule was used to numerically approximate the integral of (25), where $\Delta h$ and $h_{i}$ are known from the available data. The corresponding mid-points, $m_{i} \pm q\left(\bar{z}, h_{i}\right)$ for (17) or $m_{i} \pm h_{i}\left(1-\tau / \tau_{0}\right)$ for (19), may not correspond to the generated discrete data points, so we use linear interpolation between neighboring discrete mid-points to determine the appropriate value to use. When $m_{i}+q\left(\bar{z}, h_{i}\right)$ $>m_{\max }$ or $m_{i}-q\left(\bar{z}, h_{i}\right)<m_{\min }$, we set $\Delta t\left(m_{i} \pm q\left(\bar{z}, h_{i}\right), h_{i}\right)=0$. This will lead to artifacts at the edges of the anomaly field, in our reconstruction procedure.

The integrations in (17) or (19) are carried out $J K$ times to calculate the discrete back-projected anomaly field $\hat{n}_{1}\left(x_{j}, z_{k}\right)(j=1, \cdots, J, k=1, \cdots, K)$. We now convolve this back-projected field with $F\left(x_{j}\right)$. The filter, $F\left(x_{j}\right)$, is calculated by 
defining the following tapered function in discrete Fourier space

$$
\tilde{F}(J-j)=\tilde{F}(j)=\frac{2 \pi j}{64} \cos \left(\frac{\pi j}{J}\right) j=0, \quad \frac{J}{2} .
$$

We now take $F(j)$ as the inverse Fourier transform of $\tilde{F}(j)$. However, we find it

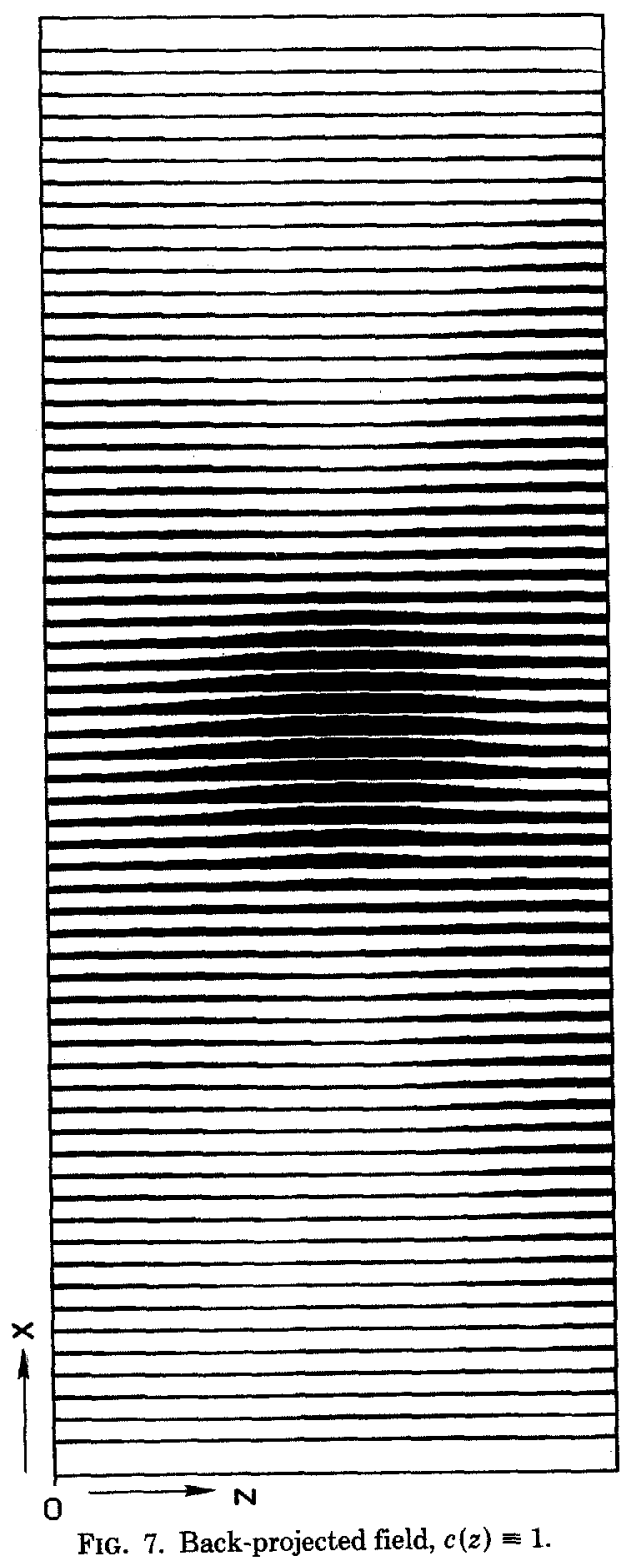

necessary to pad $\hat{n}_{1}\left(x_{j}, z\right)$ and $F(j)$ with zeros to avoid the wrap around effects from the subsequent convolution.

As we shall see, the differences between the results of using (19) or (17) are slight (at least, when viewed with out plotting format). However, (19) is much quicker 
computationally, as the amount of function evaluation involved in the integration is much less than in (17).

Numerical examples

\section{Example 1}

In this example, we consider a disk of radius 1 , with constant slowness pertur-

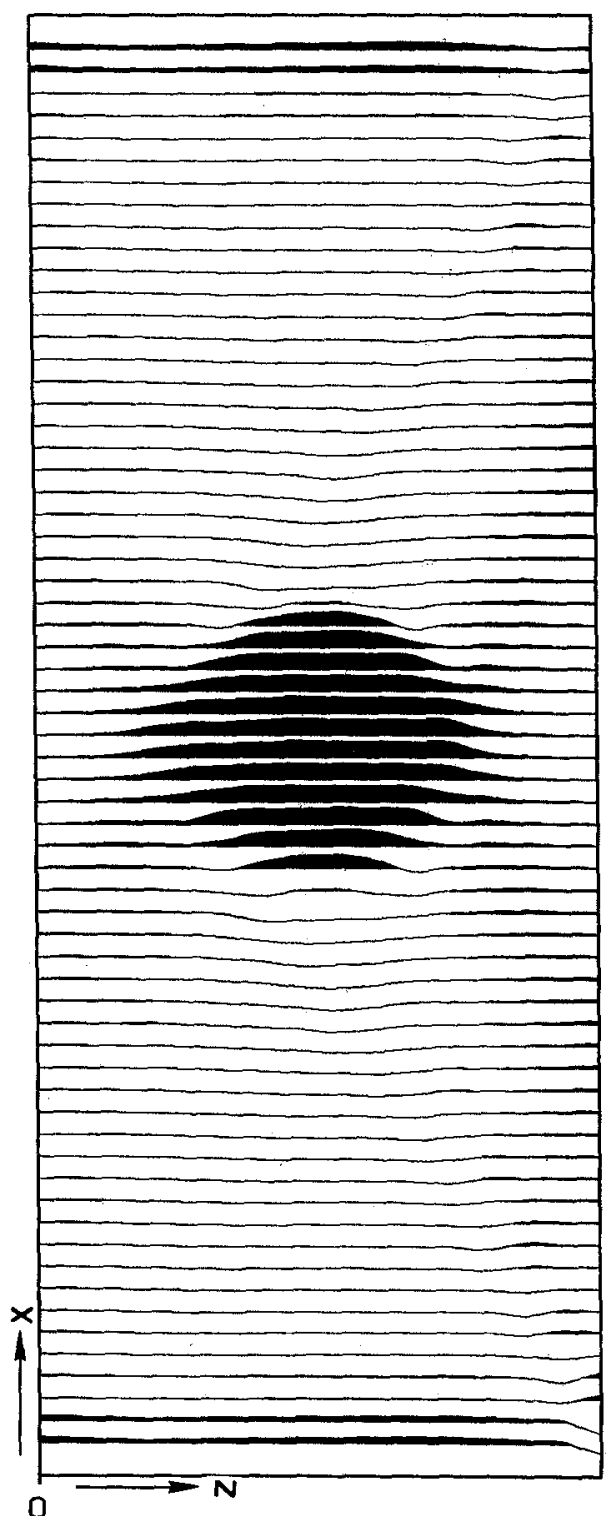

FIG. 8. Filtered back-projected field, $c(z) \equiv 1$.

bation, 1, located at the center of the field. Our minimum and maximum mid-points are for this example, $m_{\min }=-5$ and $m_{\max }=5$, and the depth of the reflector is $z_{0}=4$. The geometry for this example is shown above in Figure 5 .

Will will vary " $a$ " in $c(z)=a z+b$, and the maximum offset $h_{\text {max }}$, for different 
inversions. In all our examples, we calculate 64 mid-point positions, where the discrete mid-point positions are given by $m_{j}=m_{\min }+j\left(\left(m_{\max }-m_{\min }\right) / 63\right)(j=0$, 63). For our first inversion, we take $c(z)=1$. The offsets, $h_{k}$ are calculated from $h_{k}$ $=\frac{k}{63} 8.953(k=0,63)$. Figure 6 shows the travel-time perturbation data for this

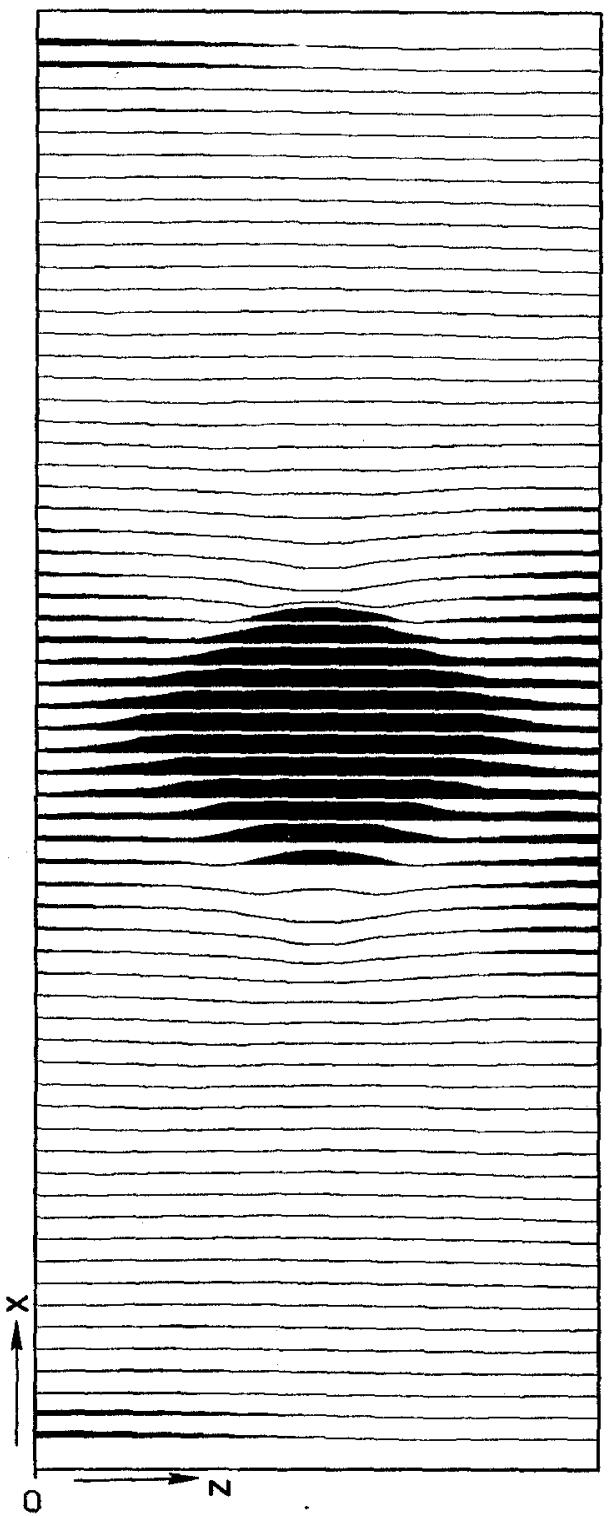

FIG. 9. Reconstructed field; $h_{\max }=4.48, c(z)=1$.

model. We note the two "arms" of data. If we had taken a point anomaly at $(\bar{m}, \bar{z})$ instead of a finite thickness disk, then the arms would be two straight lines, and the slope of these lines would give the depth to the anomaly. From equation (23)

$$
\frac{m-\bar{m}}{h}=1-\frac{\bar{z}}{z_{0}} \text {. }
$$


To invert the travel-time data, $\Delta t(m, h)$, for $n_{1}(\bar{x}, \bar{z})$, we will numerically implement (23). In Figure 7, we show the back-projection approximation to $n_{1}(x, z)$ [i.e., $F(x)$ has not yet been applied]. Here, the plot shows 64 mid-point positions and 16 depth increments $z_{k}=\frac{4 k}{15}(k=0,15)$. Figure 7 agrees qualitatively with the

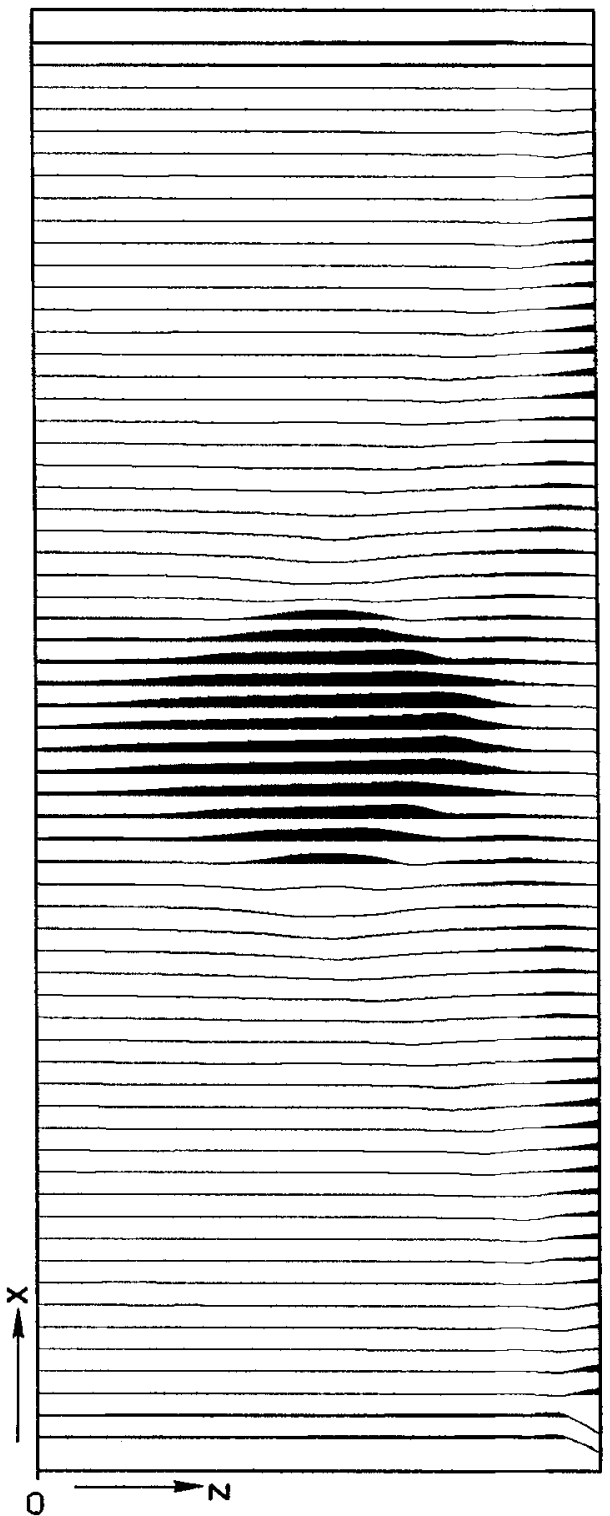

FIG. 10. Reconstructed field from (17); $h_{\max }=5.98, c(z)=0.2 z+1$.

concept of convolving the "true" symmetrized field, $\Delta n(x, z)$, with the filter $\Theta(x, z)$, described by (16a). We see immediately that a filter $F(x)$ which "kills" slow horizontal variations and amplifies shorter wavelengths will improve the image of Figure 7. The anomaly field obtained by applying $F(x)$ to the back-projected field is shown in Figure 8. We now half the offset coverage used above. The tomographically reconstructed field is shown in Figure 9. As we expect from the section on 
"Depth and the Relative Resolution," the anomaly's vertical extent is now less well resolved.

We now calculate $\Delta t(m, h)$ for the background field $c(z)=0.2 z+1$. The maximum offset we use of $h_{\max }=5.98$, and we calculate 64 increments in $m$ and $h$. Figure 10

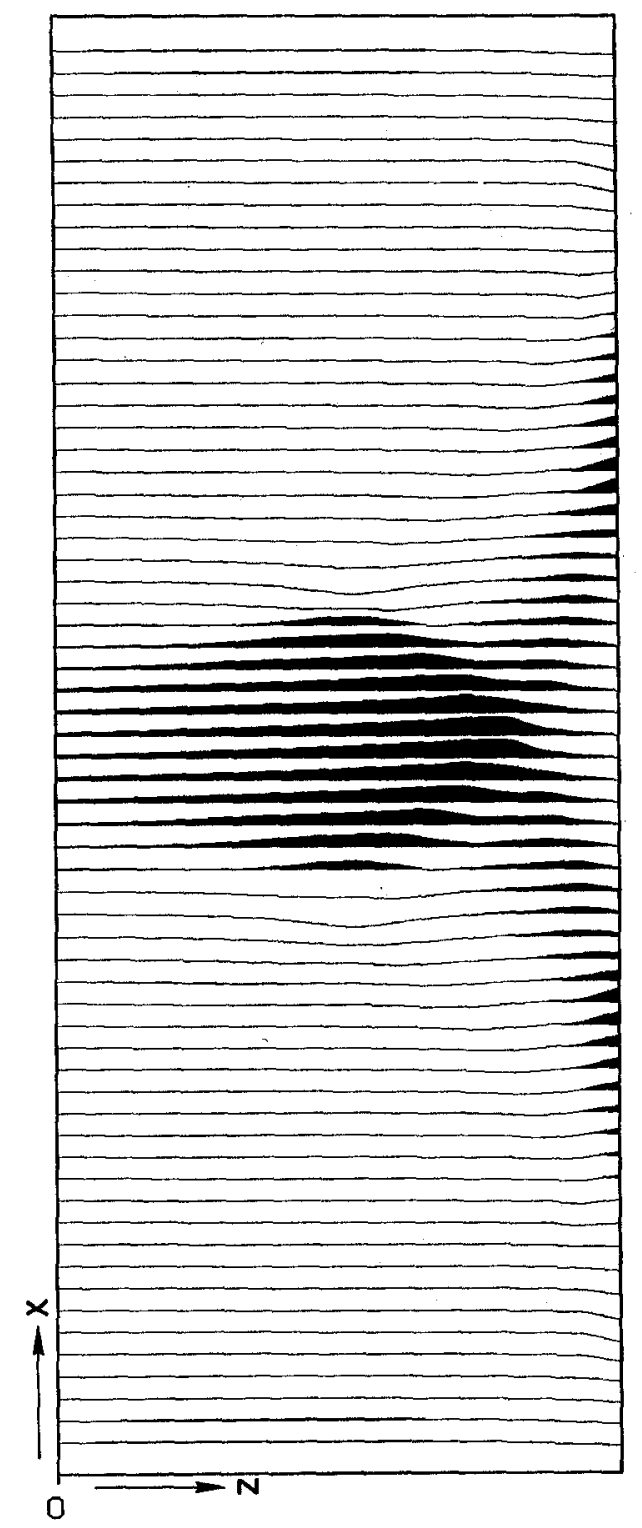

FIG. 11. Reconstructed field from (17); $h_{\max }=3.92, c(z)=z+1$.

shows the reconstructed field using formula (17). We note, that as we discussed in "Depth and the Relative Resolution," the vertical resolution of the anomaly, particularly at the top of the field, has decreased. For $c(z)=z+1, h_{\max }=3.92$, and the reconstructed field, using (17), is shown in Figure 11, and using (19), Figure 12. 
In Figure 13, we show the result of an inversion [data for $c(z)=z+1$ ] using the background velocity field to be a constant (i.e., the rays are straight). We can see from this example that the bending of the background rays has an important effect upon the inversion results.

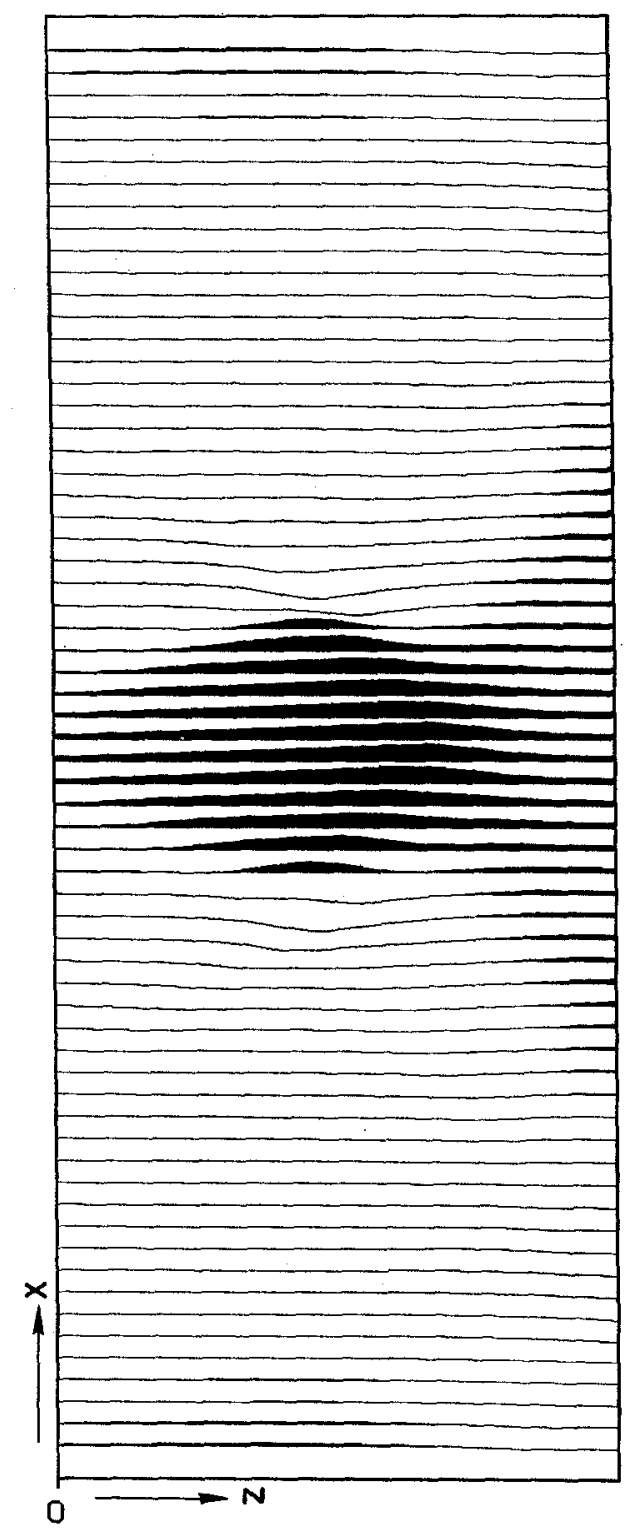

FIG. 12. Reconstructed field from (19); $h_{\max }=3.92, c(z)=z+1$.

\section{Example 2}

As we have discussed, for a background field that increases in depth, the resolution of our tomographic reconstruction improves with depth. In this example, we consider three disks of varying position, radii, and slowness perturbation strength, located 


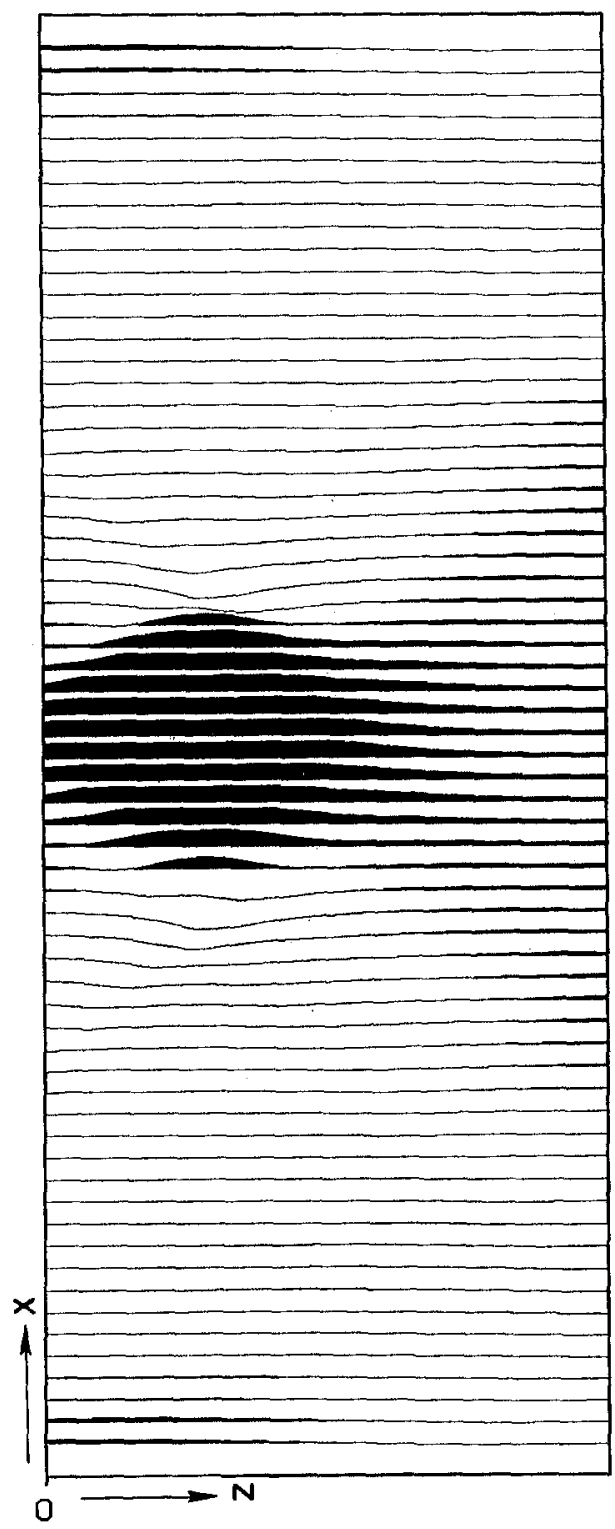

FIG. 13. Reconstructed field using $c(z)$ constant, $h_{\max }=3.92, c_{0}(z)=z+1$.

near the bottom of the layer. Some rays pass through more than one disk.

Once again, $m_{\min }=-5, m_{\max }=5$ and the reflector is at $z_{0}=4$. The disks all have their centers at depth $z=3$, with horizontal coordinates $x_{1}=-3, x_{2}=0, x_{3}=2$. The constant slowness perturbations in each are 2,1 , and 2 , respectively. Schematically, the anomaly field is shown below in Figure 14. The travel-time data, $\Delta t(m, h)$, is plotted in Figure 15. Finally, in Figure 16, a and b, we show the results of the inversion using formulas (17) and (19), respectively. 


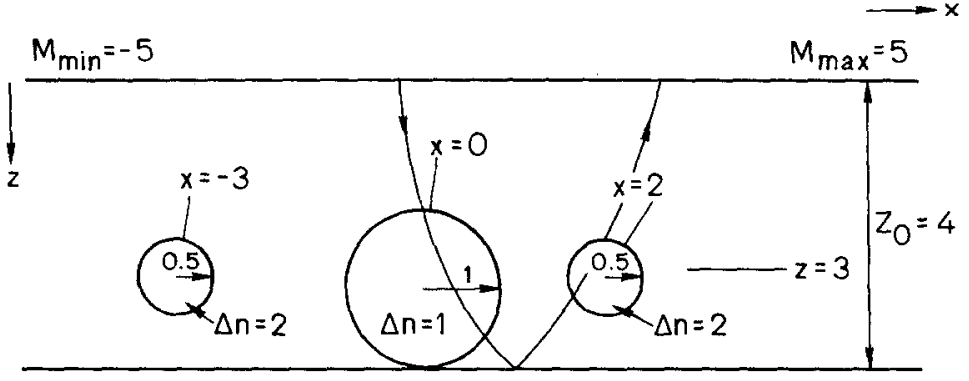

FIG. 14. Geometry for example 2.

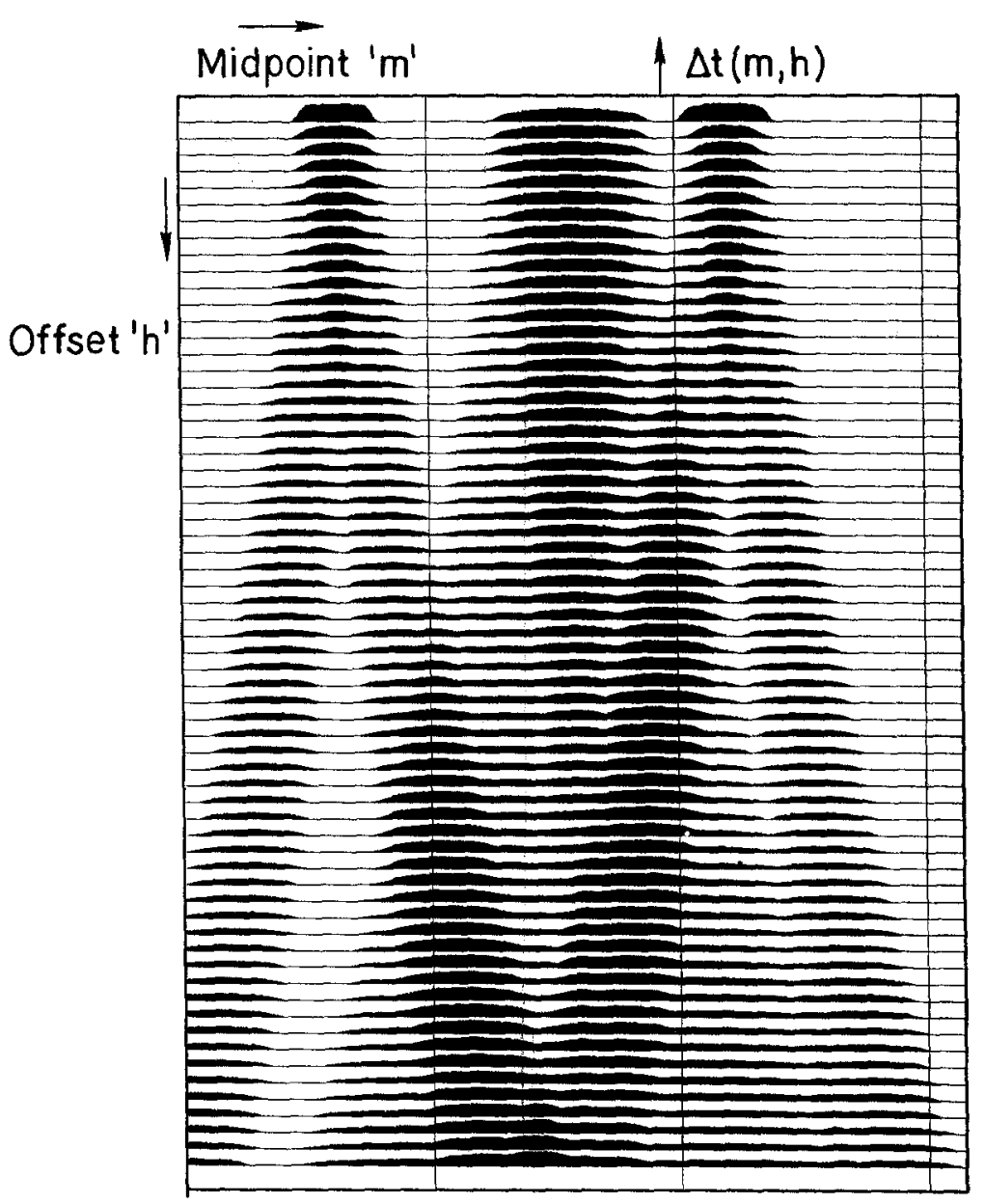

Fig. 15. Travel-Time data, $\Delta t(m, h)$, for Figure 16. 

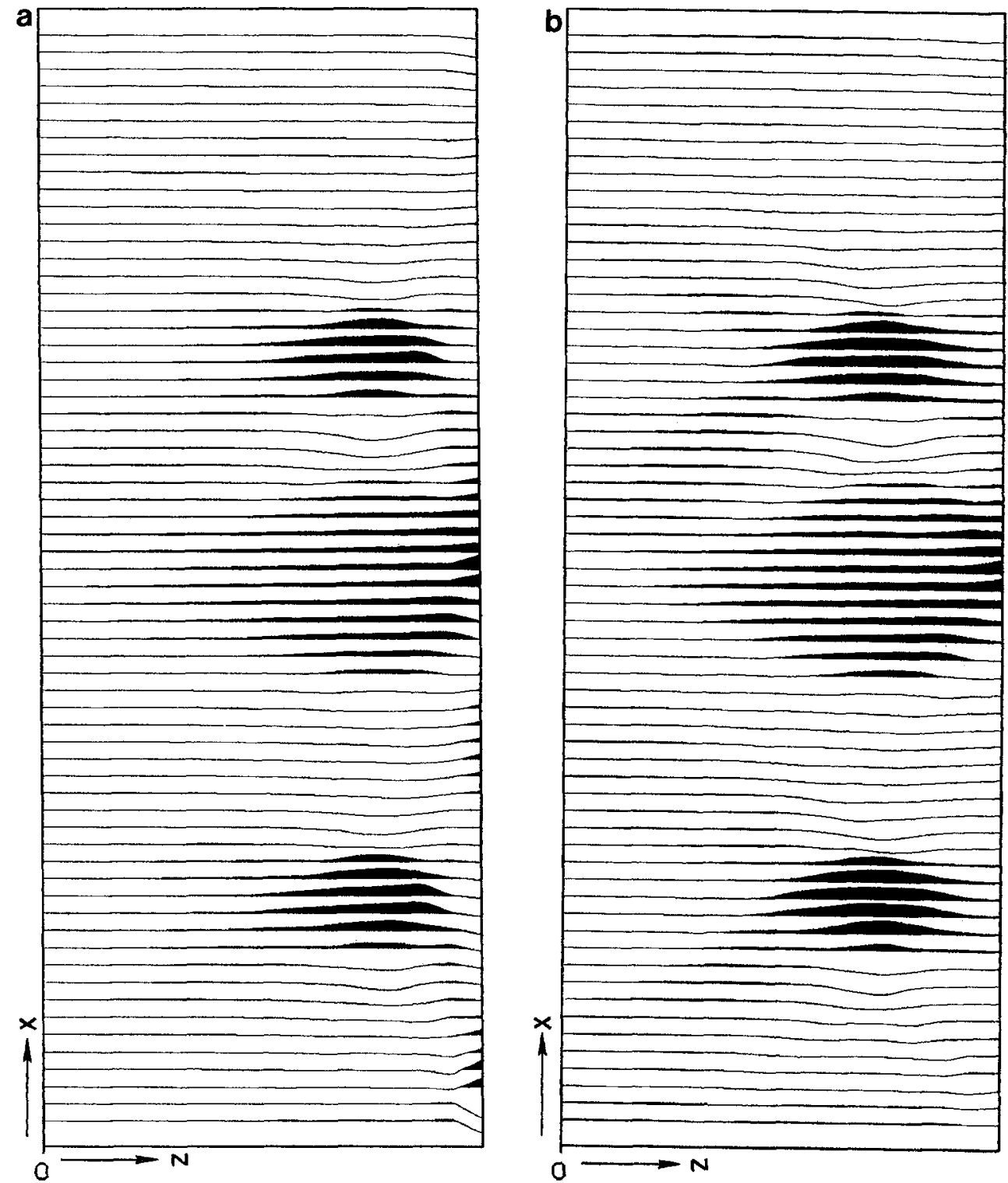

FIG. 16. (a) Inversion from (17); $c(z)=z+1, h_{\max }=3.92$. (b) Inversion from (19); $c(z)=z+1$, $h_{\max }=3.92$.

\section{CONCLUSIONS}

A simple generalized inverse radon transform (17) can be used to qualitatively reconstruct slowness anomalies with respect to a depth varying background field from observed surface reflection (flat reflector) travel-time data. Much of our analysis was based upon the assumption that the slowness anomalies were spatially localized. Thus, we could consider a local coordinate system, centered on an anomaly, and using the constant velocity problem as a model, define a local radon transform. There are certainly situations where our $a$ priori physical assumption may break down [e.g., the anomalies may not be compact, or the background ray field may have singularities (caustics)]. However, we hope that these ideas derived 
from local analysis, can also be applied to the situation of more general background media [for more work on generalized radon transforms, see Beylkin (1982)]. A simpler inversion formula, (19), based on zero offset approximations can also be effectively used for $n_{0}(\vec{x})=n_{0}(z)$.

The quality of the anomaly reconstruction at a point within the layer depends upon the "angular coverage" (i.e., the size of $\Theta_{\min }$ ) of the rays for the background field. This coverage increases with depth for a background velocity field that increases with depth. We hope to address some of the problems and extensions of our methods in future work.

\section{ACKNOWLEDGMENTS}

This paper is based upon a chapter of the first author's Ph.D. Thesis at the California Institute of Technology. This chapter was written under the supervision of the second author and Professor H. B. Keller, whom we would like to thank for his suggestions and encouragement. We would also like to thank Professor J. B. Keller at Stanford for his helpful critiques of earlier versions of this paper.

The first author (J. F.) was supported financially at the California Institute of Technology by the U.S. Department of Energy and the Natural Sciences and Engineering Research Council of Canada. At Stanford, financial support for this research was provided by the Air Force Office of Scientific Research, the Army Research Office, the Office of Naval Research, and the National Science Foundation.

\section{REFERENCES}

Beylkin, G. (1982). Generalized radon transform and its applications, Ph.D. Thesis, New York University, New York.

Fawcett, J, (1983). I. Three-dimensional ray-tracing and ray-inversion in layered media. II. Inverse scattering and curved ray tomography with applications to seismology, Ph.D. Thesis, California Institute of Technology, Pasadena, California.

Kjartansson, E. (1980). Attenuation of seismic waves in rocks, Ph.D. Thesis, Stanford University, Stanford, California.

DEPARTMENT OF MATHEMATiCS

STANFORD UNIVERSITY

STANFORD, CALIFORNIA 94305 (J.A.F.)
SEISMOLOGICAL LABORATORY

California Institute of Technology

Pasadena, California 91125 (R.W.C.)

Manuscript received 1 March 1984 